\title{
Developing an e-Strategy for the South African Astronomical Observatory Library and Information Service: A case study
}

\author{
Samantha Muriëll Bennett ${ }^{1, \star}$ and Theresa de Young ${ }^{1}$ \\ ${ }^{1}$ South African Astronomical Observatory
}

\begin{abstract}
The South African Astronomical Observatory (SAAO) Library and Information Service (LIS) forms part of the SAAO - which is a business unit of the National Research Foundation (NRF) in South Africa. Until recently each business unit had a library service that operated independently. With the formation of the NRF Knowledge Center (KC) this is set to change. Previously it was possible for a library service to conduct its processes and procedures in isolation as long as the LIS aligned with the business unit. This paper covers the study that investigated the needs and possible guidelines for an e-Strategy for the SAAO LIS that is both aligned to the broader NRF strategy but which also incorporates recommendations to upgrade the library products and services offering. The pool of Information and Communication Technology (ICT) -based products and services is growing. 'Upgrading' therefore requires that it is acknowledged that ICT provides essential infrastructure for the relevant the library. Library focused trends show continuous development. Bringing the SAAO LIS up to standard will require that these developments are continuously monitored for relevance and incorporation. This paper provides information about the SAAO LIS and its history. Both 'SWOT' and 'PESTEL' analyses were used to direct the discussion on the internal and external influences on the SAAO LIS. The analyses also provided insight into the challenges the SAAO LIS faces, the strengths that could be capitalized and the opportunities that could be exploited. The paper recommends that the SAAO LIS, before engaging in a strategic change, addresses the challenges of sustainable funding and accessibility to core resources. It is also recommends that the SAAO LIS uses its e-Strategy to 'fast track' the process of bringing it up to the standard required of world-class astronomy libraries.
\end{abstract}

\section{Introduction}

The South African Astronomical Observatory (SAAO), a National Research Foundation (NRF) facility, is the South African national resource for optical/infrared astronomy. Its primary function is to conduct fundamental research in astronomy and astrophysics. It not only provides a world-class facility that promotes astronomy and astrophysics in Southern Africa, but it also connects South African astronomical research to the rest of the world. The Library and Information Service (LIS) of the SAAO is seen as an integral part of the facility as it provides the research staff with access to essential information and tools that allow them to function as informed professionals within their community.

\footnotetext{
^e-mail: sbennett@hct.ac.ae
} 
Because the SAAO is part of the larger NRF-facility family the LIS of the SAAO also aligned its strategic actions with those of the NRF. Any strategic actions adopted by the library need to contribute also to the NRF's strategic direction and should assist in achieving the NRF's corporate goals.

This e-Strategy case study was conducted in 2014 and NRF's 2012-2015 annual performance plan ([1])was applicable, stating its vision as: "ensuring research excellence within a transformed research workforce, and a sustainable environment, not only for the benefit of the present generation but also for future generations of South Africans." In short, this vision seeks to create:

- World-class research

- A transformed society

- A sustainable environment

The plan also indicated the NRF's mission as the following: To contribute to the knowledge economy in South Africa by attaining at least one percent of global research and development (R\&D) output by 2015 .

In support of achieving its objectives the NRF adopted the following set of values:

- Passion for excellence

- World class service

- Ethics and integrity

- Respect

- People centered

- Accountability

These values highlight a passion for excellence and world class research and service through the organisation's sound ethical practices and accountability. The NRF also values its staff.

The SAAO is one of 6 reporting facilities to the NRF. It appears though that the LIS of the SAAO has not kept pace with the growth within the rest of the SAAO. It is therefore essential that the LIS re-looks at its reason for existence and that it fully and explicitly aligns with the NRF values as well as with developments within the LIS sector. It especially appears that the LIS of the SAAO has not stayed aligned with the technological developments in either the library sector or with the changes that the rest of the SAAO has experienced. It is therefore anticipated that the opportunities, brought about by changes in technology, may need to be investigated in considerable detail.

\subsection{Overview of the SAAO historic background}

The SAAO was formed in 1972 by the amalgamation of two older institutions, the Royal Observatory, Cape of Good Hope, established during 1820 and the Republic Observatory, Johannesburg, established during 1905. ([2]) "The LIS at the SAAO dates its existence to the arrival of the first Royal Astronomer in the 1820s. The SAAO LIS is now the national library for astronomy and space science. The larger collection is located in the Main Building at SAAO, Cape Town, with a smaller collection at the observing site in Sutherland. The library has a unique richness of pre-twentieth century journals with the core astronomical journals starting at volume 1." ([2]) The Observatory buildings, including the main building which hosts the library, are old and due for restoration and refurbishment. Restoration of the Observatory would be beneficial for the public and incline preservation of Astronomical heritage of South Africa. "Friends of the Cape Town Observatory" have supplied advice and are encouraging the South African Heritage Resource Agency (SAHRA) to declare the Observatory as a National Heritage Site. ([3]) 


\subsection{LIS holdings}

The LIS online catalogue was started in 1993 and since 2004 the library personnel have been engaged in retrospective online cataloguing. The online catalogue currently gives access to 9,521 records and 3,454 staff publications (compared to the card catalogue which holds just over 30,000 records). The online catalogue is currently only available from the SAAO intranet.

The library currently uses DB Text software powered by Mindex / InMagic as a library system. This system provides the necessary customisation and personalisation to fulfil the library's needs. The holdings of the library are partly catalogued. The libraries' holdings consists of journals (electronic and print), books (electronic and print), databases, atlases and catalogues, brochures, maps, photographs, plates, posters, staff publications, slides, media collections, CD's / DVD's and many historic documents. Not all of these holdings are catalogued on the library system or are in any organised form. Most of the holdings are scattered throughout the observatory buildings.

The library maintains a database of staff publications and papers produced by observers using data obtained at the SAAO. These publications are available on the library catalogue from 1998-current.

The NRF invested in DSpace, an open source repository software package used for the creation of open access repositories for scholarly and/or published digital content. DSpace could also be used as a digital archives access system when focusing on long-term storage, access and preservation of digital content. DSpace offers a mobile interface for easy accessibility as an important needed feature. The SAAO library has rights to upload its collections on DSpace to be available. However, the astronomy community's needs are very peculiar and distinct.

\subsection{Explaining the research}

The study intended to at least catalogue the library holdings at collection level. It also evaluated the library system and the value of the library content by guiding the organisation in evaluating the collection(s) hosted by the library. Furthermore, it assisted in identifying which collections were worth digitising and which collections needed to be preserved in the longer term.

The main focus of any library is to avail all collections, i.e. to be accessible as Buckland (1992:

$14)$ indicated. Accessibility for the SAAO library is twofold:

- The collections are widely spread throughout the SAAO buildings. Many of these collections are not reflected in the card catalogues and searching for the collections is a huge task

- The online catalogue is equally outdated and research staff only have access to the electronic catalogue when they are on site.

\section{Research question and sub-questions}

Although the physical library may have defects it is anticipated that, going forward, the focus should be on a hybrid library with predominantly electronic content. This case study therefore focused on developing an e-strategy for the SAAO library. The research question is therefore: Given its unique context, what would be an appropriate e-strategy for the SAAO library to follow? In support of the research question above the following sub-questions have been developed:

- What, according to literature, are the most important factors to consider when developing an estrategy for a Special Library?

- What could be learned from other astronomical libraries?

- What is the current status of the SAAO library - also within the broader NRF family? 
- What are the explicit information needs of the SAAO researchers?

- What would the implementation priorities at SAAO be (with regards to technologies, collections and staffing)?

\section{Scope and limitations of the research}

This research only focused on the SAAO library collection and current library system prior to 2015 as per the status perceived by the researcher and benchmarked with senior staff at the SAAO. Astronomical and science library needs are distinct and leave librarians with challenges as Gomez ([? ]) highlighted. However, in the scope of these challenges a comparison with world class astronomical libraries was done for benchmarking purposes. Furthermore, the study did not focus on the different user groups of researcher and astronomers, but generalised its focus to that of the SAAO. The study also provided a technology map of the current systems used prior to 2015 within the library, and made no provision to investigate new systems.

\section{Research Approach}

A qualitative approach was used for analysing and interpreting the content of documents, articles and web pages relating to the SAAO and the e-strategies of other similar institutions. A literature review was done to determine what the modern library should be. This review also highlighted best practices used in other libraries as a means of comparison. The third part of the data collection was an online survey administered to staff and students to determine what is needed to successfully implement accessibility, be it online or in hard copy.

\section{Value of the study}

This study provided useful information to the SAAO (and also the NRF) regarding the proposed 'updated' library and its collections or holdings. It aims to assist the librarian to create focus in terms of priorities, and to create order and access to the valuable and distinct information it could host online. It also provides an explicit 'key' or map as to where information and collections are stored or kept.

This is the first study for the SAAO library that focuses on the accessibility of information and the preservation of valuable information collections. Since the SAAO is the sole owner of South African astronomical data and information, giving guidance regarding the digitisation of the most valuable collections is a specific valuable aspect of this study.

This study adds value to the world of astronomy libraries as many of them ([? ]) face the same challenges such as paper based information, which is unorganised or is accumulated over decades or even centuries without having kept pace with the digital revolution and the creation of new spaces. By developing the e-strategy, it will be possible to provide focus and guidance and it will assist the SAAO library to make informed decisions regarding its online future.

\section{Obstacles during the study}

During the study a few obstacles were encountered, which need to be noted. The focus group selection was opened to be inclusive of all divisions using the library. In the end the group only consisted of those staff members who had an interest in the library and it excluded administrative staff and representation from instrumentation department. 
Even though the survey was sent to a sample of friends and colleagues to test for errors, it still forced people to answer questions that were not relevant to them. This was discovered and dealt with during the data analysis. Astronomy library literature is limited and left a gap in finding information relevant to the subject field. General library information needed to be used as guideline. The internal organisational culture was another hurdle and forced the researcher to use softer approaches in getting information.

\section{Recommendations}

ICT has become an important driver to the library. Due to the ever changing environment the library finds itself in, it has become clear that the pool of IT services and products is growing. For the library to best utilise these systems they have to ensure that the support supplied by these systems share the goals of the strategic plan. To best interpret the services required the library needed an e-strategy which was provided as an end result of the research.

\section{Update on the current status}

The recommendations of the study have been adopted, although funding has been the main concern in implementing them. These recommendations have been categorized into five main groups for ease of reporting on the current status:

\subsection{Expand access to information}

Retrospective online cataloguing, to ensure that most of the items in the card catalogue are captured on the library management system, is making good progress although it is slow and time consuming. It takes a lot of effort and is not a dedicated project but rather done whenever possible. Accessibility to catalogue records and ultimately actual library items is crucial for improving the library and information service at the SAAO.

Collections are being efficiently organised and archived by very enthusiastic "Friends of the Cape Town Observatory". Many of the items that were stashed away in different locations at the observatory have been worked through, weeded, categorised and preserved where necessary. During this clean-up exercise, many valuable items were found, among them two rare books. Access to these collections are of value to historical researchers, many of whom approach the SAAO library for information.

In order to provide access to cutting edge journal papers within astronomy, the SAAO needs to become involved in consortia deals that would be a benefit, providing wider access to information. The SAAO is a member of the SCOAP3 partnership that negotiates lower subscription fees on journals. SCOAP3 journals are open for any scientist to publish in without the worry of costs.

The National Research Foundation (NRF) is currently embarking on an initiative to preserve the heritage collection at the SAAO and has purchased major digitization equipment to accomplish the scanning of these items and then the preservation thereof. Digitisation of collections is scheduled to be complete by 2019. Funding has been secured to revamp the library by building a mezzanine floor in order to alleviate some of the space limitations in terms of shelving of library material that is being used. All of this is in preparation for the bicentennial of the establishment of the observatory at the Cape of Good Hope, to be held on 20 October 2020. This will also enhance accessibility to the collections that have been scattered in buildings around the observatory and that are not easily available to staff and the public. 


\subsection{A centralised library system}

The study recommends the standardisation of library and information services within the NRF. There is a need to acquire a library management system that is more affordable and appropriate for the SAAO as well as other NRF facilities. The study also proposes a centralised library system within the NRF.

Conversations have started in this direction keeping in mind the benefits for the NRF in terms of cost and sharing of and access to all collections. Important for centralisation is the roll of NRF head office staff in taking the lead. Major changes with the staffing at the NRF appears to be the main obstacle to this; the lead can only be taken once structures are stable.

Shared e-services have commenced in 2016 with the centralised procurement of journals for all NRF facilities via a multi-year tender contract. This has proved to be a burden on the SAAO library budget as it had become more expensive than envisioned.

\subsection{Develop ICT infrastructure}

ICT infrastructure development hinges very much on availability of funding, whilst procedures for standardising processes, systems and tools are within reach as long as there is strong championing of these initiatives. Collaboration efforts together with various library partners within the SAAO will be considered and through collective efforts a stable infrastructure could be maintained. However, the standardisation of ICT within the NRF as a whole is a lot more tricky and very much dependent, once again, on head office leadership. The central point of access to one website that is recommended by the study as well as the unified use of cloud based services and device neutral platforms demands tight cooperation across the NRF including both head office and the facilities.

\subsection{Provide research support}

Research support, one of the key performance areas of the library and information service at the Observatory is recognised at the SAAO, with the librarian reporting directly to the Head of Astronomy. Along with research support comes the digital curation of intellectual work at the observatory. The NRF has taken the lead in this, adopting DSpace-CRIS open source module as the platform for managing data within the organisation including the setting up and maintaining of repositories at every one of its institutions, as well as making metrics (altmetrics) accessible, all of which are of interest in terms of research support. The NRF has also issued a 'Statement on Open Access to Research Publications from the National Research Foundation (NRF)-Funded Research' in January 2015, recognising the importance of open access to scientific research and instructing its researchers to deposit their final peer-reviewed accepted papers into the institutional repository with an embargo period attached.

\subsection{Collaborate with astronomy partners}

There is a dire need for more interaction and collaboration with astronomy library services partners across South Africa and Africa. Librarians from this continent need to be more engaged in astronomy internationally, making use of the networking opportunities to learn and share information, thus improving services to researchers. The SAAO, together with the Hartebeesport Radio Astronomy Observatory, will endeavour to encourage astronomy librarians across Africa to participate in meetings such as those organised through global initiatives such as the LISA conference. 


\section{Conclusion}

The process of change may take some time as the SAAO LIS needs to first address the challenges of funding as well as accessibility limitations. It is however essential that priorities are set and a strategic plan is developed so that the process of LIS rejuvenation can commence.

\section{References}

[1] NRF: Annual performance plan 2012-2015, (2014), http://www.nrf.ac.za/document/annualperformance-plan-2012-2015

[2] SAAO: History of the South African Astronomical Observatory, (2014), http://www.saao.ac.za/about/history/

[3] Friends of the Observatory, (2014), https://friends.saao.ac.za/

[4] M. Gomez, IAC library: Some Challenges. in 21st Century Astronomy Librarianship, From New Ideas to Action, edited by Eva Isaksson, Jill Lagerstrom, András Holl, and Nirupama Bawdekar (2010), Vol. 433 of Astronomical Society of the Pacific Conference Series, p. 47-54. Library and Information Services in Astronomy VI. ASP Conference Series, p. 47-54. 